\title{
CD73 expression defines immune, molecular, and clinicopathological subgroups of lung adenocarcinoma
}

\author{
Pedro Rocha ${ }^{1,2} \cdot$ Ruth Salazar $^{1}$. Jiexin Zhang ${ }^{3}$. Debora Ledesma ${ }^{1}$. Jose L. Solorzano ${ }^{1}$ Barbara Mino ${ }^{1}$. \\ Pamela Villalobos ${ }^{1} \cdot$ Hitoshi Dejima $^{1} \cdot$ Dzifa Y. Douse $^{1} \cdot$ Lixia Diao $^{3} \cdot$ Kyle Gregory Mitchell $^{4} \cdot$ Xiuning Le $^{4}$. \\ Jianjun Zhang ${ }^{4} \cdot$ Annikka Weissferdt $^{6} \cdot$ Edwin Parra-Cuentas $^{1} \cdot$ Tina Cascone $^{4} \cdot$ David C. Rice $^{5} \cdot$ Boris Sepesi $^{4}$. \\ Neda Kalhor ${ }^{6}$. Cesar Moran ${ }^{6}$. Ara Vaporciyan ${ }^{5} \cdot$ John Heymach $^{4}$. Don L. Gibbons ${ }^{4} \cdot$ J. Jack Lee $^{3} \cdot$ Humam Kadara $^{1}$. \\ Ignacio Wistuba ${ }^{1,4} \cdot$ Carmen Behrens $^{4}$. Luisa Maren Solis ${ }^{1}$ (])
}

Received: 15 June 2020 / Accepted: 6 December 2020 / Published online: 8 January 2021

(c) The Author(s) 2021, corrected publication 2021

\begin{abstract}
Introduction CD73 is a membrane-bound enzyme crucial in adenosine generation. The adenosinergic pathway plays a critical role in immunosuppression and in anti-tumor effects of immune checkpoint inhibitors (ICI). Here, we interrogated CD73 expression in a richly annotated cohort of human lung adenocarcinoma (LUAD) and its association with clinicopathological, immune, and molecular features to better understand the role of this immune marker in LUAD pathobiology.

Materials and methods Protein expression of CD73 was evaluated by immunohistochemistry in 106 archived LUADs from patients that underwent surgical treatment without neoadjuvant therapy. Total CD73 $(\mathrm{T}+)$ was calculated as the average of luminal $(\mathrm{L}+)$ and basolateral $(\mathrm{BL}+)$ percentage membrane expression scores for each LUAD and was used to classify tumors into three groups based on the extent of T CD73 expression (high, low, and negative).

Results $C D 73$ expression was significantly and progressively increased across normal-appearing lung tissue, adenomatous atypical hyperplasia, adenocarcinoma in situ, minimally invasive adenocarcinoma, and LUAD. In LUAD, BL CD73 expression was associated with an increase in PD-L1 expression in tumor cells and increase of tumor-associated immune cells. Stratification of LUADs based on T CD73 extent also revealed that tumors with high expression of this enzyme overall exhibited significantly elevated immune infiltration and PD-L1 protein expression. Immune profiling demonstrated that $\mathrm{T}$-cell inflammation and adenosine signatures were significantly higher in CD73-expressing lung adenocarcinomas relative to those lacking CD73.

Conclusion Our study suggests that higher CD73 expression is associated with an overall augmented host immune response, suggesting potential implications in the immune pathobiology of early stage lung adenocarcinoma. Our findings warrant further studies to explore the role of CD73 in immunotherapeutic response of LUAD.
\end{abstract}

Keywords CD73 $\cdot$ Lung adenocarcinoma $\cdot$ Immune profiling $\cdot$ Adenosinergic pathway $\cdot$ PD-L1

$\begin{array}{ll}\text { Abbreviations } \\ \text { NSCLC } & \text { Non-small cell lung cancer } \\ \text { LUADs } & \text { Lung adenocarcinomas } \\ \mathrm{T} & \text { Total }\end{array}$

Pedro Rocha and Ruth Salazar have contributed equally to this work.

Luisa Maren Solis

lmsolis@mdanderson.org

Extended author information available on the last page of the article

$\begin{array}{ll}\text { BL } & \text { Basolateral } \\ \text { L } & \text { Luminal } \\ \text { TH } & \text { Total high group } \\ \text { TL } & \text { Total low group } \\ \text { TN } & \text { Total negative group } \\ \text { TAICs } & \text { Tumor-associated immune cells. } \\ \text { ICI } & \text { Immune checkpoint inhibitors } \\ \text { PD-1 } & \text { Programmed death 1 } \\ \text { PD-L1 } & \text { Programmed death-Ligand 1 } \\ \text { CTLA-4 } & \text { Cytotoxic T lymphocyte-associated 4 } \\ \text { NK } & \text { Natural killer } \\ \text { TME } & \text { Tumor microenvironment }\end{array}$


FFPE Formalin-fixed paraffin-embedded

MCs Malignant cells

\section{Introduction}

Despite significant improvements in treatment, lung cancer remains the leading cause of cancer-related deaths worldwide [1]. Immune checkpoint inhibitors (ICI), as a single agent or in combination with chemotherapy, are increasingly becoming the standard treatment for non-small cell lung cancer (NSCLC), including advanced-stage lung adenocarcinoma (LUAD) [2-5]. Recent studies have shed light on the clinical value of immunotherapy for earlier stage lung tumors including in the neoadjuvant and adjuvant settings $[6,7]$. Yet, a limited fraction of NSCLC patients respond to immune checkpoint blockade consisting of anti-PD-1/ PD-L1 (Programmed death 1/Programmed death-ligand 1) and CTLA-4 (Cytotoxic T Lymphocyte-associated 4); perhaps warranting the need for other combinatorial immunotherapeutic regimens to potentiate anti-tumor effects of ICI.

Adenosine is generated in the tumor microenvironment owing mainly to the degradation of extracellular ATP [8-10] and NAD + [11]. Several ectonucleotidases tightly control levels of ATP and Adenosine, such as CD38, CD39, and CD73; among them, CD73 irreversibly converts AMP to Adenosine and was suggested as the rate-limiting enzyme for adenosine formation [12]. Increased adenosine levels permit an immune-tolerant tumor microenvironment by regulating the functions of immune and inflammatory cells such as macrophages, dendritic cells, myeloid-derived suppressor cells, T cells, and natural killer (NK) cells [13]. Adenosine also regulates cancer growth and dissemination by interfering with cell proliferation, apoptosis, and angiogenesis via adenosine receptors expressed on cancer cells and endothelial cells [14-16].

Tumor microenvironment (TME) immunosuppression has emerged as a sentinel mechanism in lung cancer progression and, thus, a viable phenotypic target for treatment [17, 18]. In this context, numerous therapeutic approaches are currently under development with the goal of skewing the TME toward an immune effective phenotype [19]. More recently, in preclinical studies, agents that target the adenosine pathway, including anti-CD73 antibodies and adenosine A2A receptor antagonists, were shown to also attenuate immunosuppression [20, 21].

While CD73 expression in LUAD was noted previously [22], the association of this immune enzyme mediator of the adenosine pathway with other relevant clinical biomarkers such as PD-L1, immune infiltrates, and tumor mutation burden remains unknown. We surmised that understanding the contextual expression patterns of CD73 in LUAD can help us better understand the role of the adenosine pathway in
NSCLC and in the immune pathobiology of this malignancy. Here, we sought to characterize the immunohistochemical expression of CD73 in a richly annotated cohort of early stage LUADs in association with various clinicopathological, molecular, immune features, and other markers involved in adenosine generation. We demonstrate that the extent of CD73 expression in malignant cells (MCs) defines groups of LUADs with distinct immune profiles and that thus may guide future personalized immunotherapeutic strategies.

\section{Materials and methods}

\section{Patient samples}

We first interrogated CD73 RNA expression in a set of 83 FFPE specimens from 50 patients representing different lesions in the sequence of LUAD pathogenesis including normal-appearing lung tissue $(n=38)$, adenomatous atypical hyperplasia (AAH; $n=9$ ), adenocarcinoma in situ (AIS; $n=11$ ), minimally invasive adenocarcinoma (MIA; $n=21$ ), as well as invasive adenocarcinoma $(n=4)$, and that were profiled using the nCounter, PanCancer Immune Profiling Panel (NanoString Technologies) (Supplementary Fig. 1) in the manner described previously [23]. To determine associations of CD73 and LUAD clinicopathological, molecular, and immune features, we studied a cohort of LUADs $(n=106)$ from patients with early stage (stages I-III) disease that underwent surgical treatment without neoadjuvant therapy between Feb 1999 and 2012 at The University of Texas MD Anderson Cancer Center (MD Anderson; Houston, TX, USA). This study was approved by the MD Anderson Institutional Review Board and was conducted according to the principles of the Helsinki Declaration. Formalin-fixed paraffin-embedded (FFPE) LUADs tissue was placed in a tissue microarray (TMA); the tumor samples were selected based on the availability of FFPE tissue blocks; three $1 \mathrm{~mm}$-diameter cores that included tissue from the center, intermediate, and peripheral areas of the tumor were used for the TMA, as previously described [24]. Detailed clinicopathological information, including demographics, smoking history, pathologic tumor-node-metastasis stage (staging system from the 8th American Joint Committee on Cancer) [25], histological patterns, and overall and recurrence-free survival were available for all cases and are summarized in Table 1. Briefly, the median age in this cohort was 65 years (range $41-84$ ), with ever smokers representing $86 \%$ of patients included, and with a median follow-up of 86 months. Histological growth patterns were categorized as any-solid and non-solid based on the presence of any observed solid growth pattern found [26]. Mutational status of key driver genes, including KRAS, EGFR, STK11, TP53, and mutation burden derived from whole-exome sequencing [27] or Sanger sequencing data, 
Table 1 Clinicopathological and molecular characteristics of LUAD patients studied $(N=106)$

\begin{tabular}{|c|c|}
\hline Characteristic & $N(\%)$ \\
\hline \multicolumn{2}{|l|}{ Age } \\
\hline Median (range) & $65(41-84)$ \\
\hline \multicolumn{2}{|l|}{ Sex } \\
\hline Female & $52(49 \%)$ \\
\hline Male & $54(51 \%)$ \\
\hline \multicolumn{2}{|l|}{ Smoking history } \\
\hline Never & $15(14 \%)$ \\
\hline Current/former & $91(86 \%)$ \\
\hline \multicolumn{2}{|l|}{ TNM 8th edition } \\
\hline I & $58(55 \%)$ \\
\hline II & $26(25 \%)$ \\
\hline III & $22(20 \%)$ \\
\hline \multicolumn{2}{|l|}{ Pathological T (8th) } \\
\hline $\mathrm{pT} 1 \mathrm{a}-\mathrm{pT} 2 \mathrm{a}$ & $70(66 \%)$ \\
\hline $\mathrm{pT} 2 \mathrm{~b}-\mathrm{T} 4$ & $36(34 \%)$ \\
\hline \multicolumn{2}{|l|}{ Pathological N (8th) } \\
\hline N0 & $78(74 \%)$ \\
\hline N1 & $20(19 \%)$ \\
\hline $\mathrm{N} 2$ & $8(7 \%)$ \\
\hline \multicolumn{2}{|l|}{ Histologic pattern } \\
\hline Any-solid & $46(43 \%)$ \\
\hline Non-solid & $60(57 \%)$ \\
\hline \multicolumn{2}{|l|}{ Molecular characteristics } \\
\hline$E G F R$ mutated & $15(15 \%)$ \\
\hline$E G F R$ wild type & $85(85 \%)$ \\
\hline$K R A S$ mutated & $26(25 \%)$ \\
\hline$K R A S$ wild type & $77(75 \%)$ \\
\hline TP53 mutated & $27(43 \%)$ \\
\hline TP53 wild type & $36(57 \%)$ \\
\hline STK11 mutated & $7(11 \%)$ \\
\hline STK11 wild type & $56(89 \%)$ \\
\hline \multicolumn{2}{|c|}{ Mutation burden (number of mutations) } \\
\hline Median (range) & $145(2-993)$ \\
\hline Overall survival (median) & 108.9 months \\
\hline Death & 59 \\
\hline Alive & 47 \\
\hline Recurrence-free survival (median) & 117.2 months \\
\hline Recurrence & 49 \\
\hline No recurrence & 57 \\
\hline
\end{tabular}

were available in a subset of the cases (Table 1). Also, in a subset of this cohort $(n=65)$, next-generation sequencing RNA-based data using HTG EdgeSeq Precision ImmunoOncology panel were employed to examine associations between CD73, CD38, and CD39 expression in LUAD and immune gene expression signatures [28-34] (SupplementaryTable 1), CD73 gene expression and its protein product by IHC.

\section{Immunohistochemistry staining}

We performed immune histochemistry (IHC) to detect the protein expression of CD73 (D7F9A), and CD39 (EPR20461). Antibody optimization of CD73 and CD39 was performed using tonsil tissue as control and multiple tumor specimens (including non-small cell lung carcinoma among others) to reach an optimal signal to noise ratio that can permit specific evaluation of cellular and subcellular expression patterns. Validation of the IHC assay included evaluation of FFPE lung cancer cell line pellets available with known mRNA expression of $C D 73$ and $C D 39$ (Supplementary Fig. 2). PD-L1 (E1L3N) and CD38 (SPC32) antibody immunohistochemistry validation, staining, and pathology evaluation were previously reported by our team [35]. The immunohistochemistry protocol is briefly described: tissue sections $(4 \mu \mathrm{m})$ were stained in a Leica Bond Max automated stainer (Leica Biosystems Nussloch $\mathrm{GmbH}$ ). The tissue sections were deparaffinized and rehydrated following the Leica Bond protocol. Antigen retrieval was performed for 20 min with Bond Solution \#2 (Leica Biosystems, equivalent EDTA, pH 9.0) or Bond Solution \# 1 (Leica Biosystems, equivalent Citrate Buffer, pH6). Primary antibodies were incubated for $15 \mathrm{~min}$ at room temperature and detected using the Bond Polymer Refine Detection kit (Leica Biosystems) with DAB as chromogen. The slides were counterstained with hematoxylin, dehydrated, and cover-slipped. Antibody clones and their vendor information as well as dilution and antigen retrieval conditions are summarized in SupplementaryTable 2.

\section{Immunohistochemistry scoring}

Immunohistochemistry expression levels of CD73, was evaluated in malignant cells (MCs) by two pathologists (RS and LS), using standard microscopy. The percentage of MCs with any membrane CD73 expression was estimated. Basolateral (BL) (cell membrane not adjacent to luminal spaces) and luminal (L) membrane (cell membrane facing luminal spaces) expression levels of CD73 were separately scored when evaluable (Fig. 1). To determine total CD73 (T) expression in MCs, the average of BL and L scores was computed. Tumors were categorized as CD73 positive $(\mathrm{T}+, \mathrm{BL}+$ or $\mathrm{L}+$,$) based on the presence of any mem-$ brane expression in $>1 \%$ of MCs. LUADs were stratified into three groups based on the extent of CD73 expression: 'T Negative tumors' (TN), $\leq 1 \%, n=27$; 'T Low group' (TL), $<55 \%>1 \%, n=53$, and 'T High group' (TH), $\geq$ to $55 \%, n=26$ ). Lower quartile of CD73 percentage in malignant cells was used as cutoff for T Negative group, while the upper quartile was used as cutoff for T High group (Supplementary Fig. 3a). 


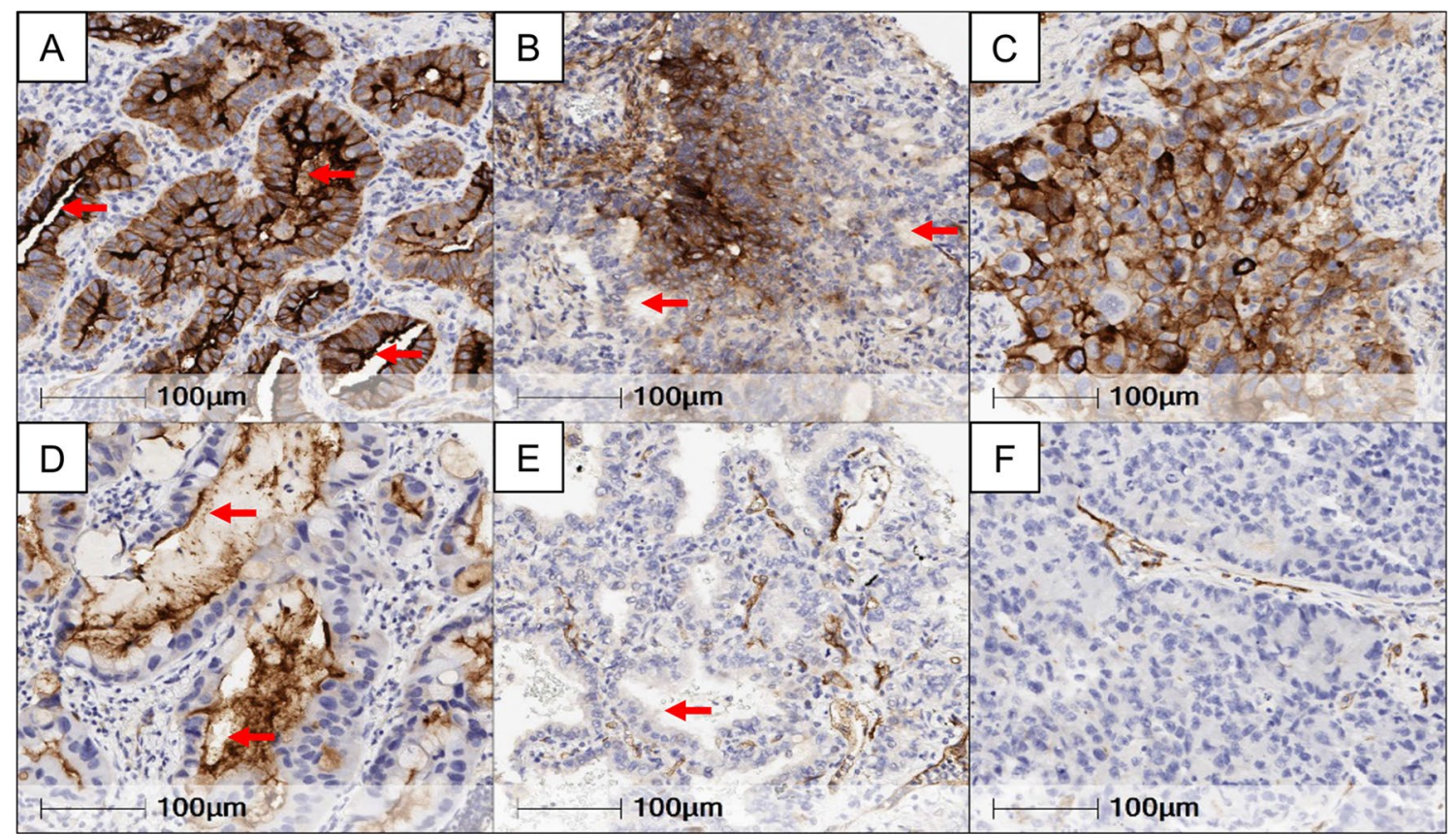

Fig. 1 Immunohistochemical expression and localization of CD73 in resectable lung adenocarcinoma. Representative microphotographs showing different patterns of CD73 expression in the luminal and/ or basolateral membrane of LUAD. a Luminal and basolateral membrane expression. b Basolateral membrane expression and with no immunoreactivity in the luminal compartment. c Basolateral mem- brane expression, with the absence of a lumen for evaluation. d Luminal membrane expression and with no immunoreactivity in the basolateral compartment. e Absence of expression in both the luminal and basolateral membranes. f No expression in the basolateral membrane and absence of a lumen. Red arrows indicate luminal membranes
Membrane or cytoplasmic CD39 expression in malignant cells was evaluated by two pathologists (DL and LS); since expression in malignant cells was not observed in any sample (0/95), expression levels of CD39 were evaluated in tumor stromal cells using digital image analysis supervised by a pathologist (DL). Briefly, the IHC-stained TMA slides were scanned using Aperio AT2 scanner (Leica Biosystem) at 20x. The digital images were visualized and analyzed with the HALO (IndicaLabs) software. A pathologist selected tumor stroma areas in each TMA core and applied algorithms to detect positive cells with cytoplasm or membrane expression of these markers; the results were expressed as cell densities $(\mathrm{n} / \mathrm{mm} 2)$ of the whole tumor stroma area analyzed; necrosis and artifacts were not included in the analysis.

Membrane PD-L1 was evaluated by two pathologists (DL and LS) as percentage of MCs with positive expression based on the International Association for the Study of Lung Cancer (IASLC) guidelines [36]. CD38 IHC expression annotated data included the evaluation in MCs and in tumor stromal cells, and were previously published by our team [35].

\section{Digital image analysis of tumor-associated immune cells}

Immunohistochemistry and digital image analysis previously performed for a subset of LUADs $(n=94)$, included the analysis of cell densities of tumor-associated immune cells (TAICs): CD3 + (T cells), CD4 + (helper T cell), $\mathrm{CD} 8+$ (cytotoxic $\mathrm{T}$ cell $), \mathrm{CD} 57+(\mathrm{NK}$ cells $)$, granzyme $\mathrm{B}+(\mathrm{NK} /$ cytotoxic $\mathrm{T}$ cells $), \mathrm{CD} 45 \mathrm{RO}+($ memory $\mathrm{T}$ cell $)$, PD-1 +, FOXP3 + (regulatory T cell), and CD68 + (tumorassociated macrophages). The IHC methodology and image data analysis were performed as previously reported by our group [37, 38].

\section{Statistical analysis}

CD73 mRNA expression across normal, preneoplastic, and malignant issues in the sequence of LUAD development was statistically determined using ANOVA and Benjamini-Hochberg correction. Targeted immune gene expression data were first median-normalized and then $\log 2$ transformed for further analysis. Scores of previously curated 
immune gene signatures were calculated by computing average expression of genes within each signature. To determine the association of categorical CD73 expression $(\mathrm{T}+, \mathrm{BL}+$, and $\mathrm{L}+$, and $\mathrm{T}$ high, $\mathrm{T}$ Low, and $\mathrm{T}$ negative) with clinicopathological characteristics, we used Fisher's exact test, as appropriate for categorical data. To test association between continuous and categorical variables, Wilcoxon signed-rank test and Kruskal-Wallis were applied for categorical variables with two levels or more than two levels, respectively. To correlate the association between continuous CD73 expression, immune markers, and immune signatures, we used Spearman's rank correlation, and scatterplots. For survival analysis, we used Cox proportional-hazard model with CD73 expression as continuous and categorical variables separately. Heat maps of CD73 expression and tumor-associated immune cells and PD-L1 expression were generated after normalizing values for better visualization of data.

\section{Results}

\section{Membrane expression patterns of CD73 and their association with clinicopathological features and immune biomarkers}

We first interrogated expression patterns of CD73 in the pathogenesis of LUAD. We evaluated the expression of CD73 mRNA in a series of premalignant lesions, along with malignant tumors, representing the sequence of pathogenesis of LUAD (83 specimens from 50 patients). We found that $C D 73$ expression was significantly and progressively increased across normal-appearing lung tissue, AAH, AIS, MIA, and adenocarcinoma ( $\mathrm{p}<0.0001$; Supplementary Fig. 1). These findings prompted us to comprehensively examine CD73 protein expression patterns in a larger cohort of early stage LUAD.

In our cohort of resectable early stage LUAD, immunohistochemistry evaluation revealed a positive total $(\mathrm{T}+)$ CD73 expression (>1\%) in 75\% (79/106) of LUADs. Positive basolateral $(\mathrm{BL}+)$ expression was found in $60 \%$ (68/106); positive luminal $(\mathrm{L}+)$ was present in $83 \%$ (60/72) of LUADs that had luminal structures in the TMA cores. Positive correlation was found between $\mathrm{BL}$ and $\mathrm{L}$ CD73 expression $(r=0.49 p=0.0042)$. Detailed information on $\mathrm{L}$ and $\mathrm{BL}$ co-expression is presented in Supplementary Table 3. Associations of CD73 expression with clinicopathological characteristics are shown in Supplementary Table 4. In our cohort, never smokers showed higher rates of $\mathrm{T}+\mathrm{CD} 73$ expression $(15 / 15,100 \%)$ compared to ever smoker patients $(64 / 91,70 \%)(p=0.0107)$. Tumors with any-solid histological pattern were associated with lower frequencies of T + CD73 $(p=0.0243)$. LUADs from female patients had higher frequency of BL + CD73 $(p=0.0268)$. We did not find correlations between CD73 expression and survival outcomes (data not shown). T and BL expression levels showed positive correlation with most immune biomarkers evaluated by IHC (Fig. 2a and Supplementary Fig. 4a). Specifically, CD73 T and BL expression levels correlated with higher PD-L1 expression $(r=0.38, p=0.0013$ and, $r=0.44, p<0.0001$, respectively) (Fig. 2b) and with higher densities of CD3, CD4, CD8, CD45RO, CD68, PD-1, FOXP3, and Granzyme B positive cells (all $p<0.05$; Fig. $2 \mathrm{c}$, and Supplementary Fig. 4a). L CD73 levels positively correlated with CD3 and CD4 cell densities (Supplementary Fig. 4b).

In a subset of patients $(N=65)$, we performed RNAsequencing-based analysis of immune genes and signatures using the HTG EdgeSeq platform. Corroborating our immunohistochemical analyses, BL CD73 gene expression was positively associated with increased expression of T-cell inflammation $(p=0.013)$ and adenosine signatures $(p=0.035)$ (Fig. 2d).

\section{CD73 expression defines subgroups of LUADs with disparate clinicopathological and immune features}

We then defined groups of LUADs (designated as high, low, and negative) based on the extent of CD73 membrane expression. CD73 IHC expression across the three groups significantly and positively correlated with its RNA counterpart ( $p<0.0001$, Supplementary Fig. 3b). Based on the extent of CD73 expression, we stratified our cohort into three groups: 'T Negative tumors' (TN), $\leq 1 \%, n=27$; ' $\mathrm{T}$ Low group' (TL), $>1 \%$ and $<55 \%, n=53$; and 'T High group' (TH), $\geq$ to $55 \%, n=26)$. $\mathrm{L}+$ and $\mathrm{BL}+$ expressions in these groups are shown in Supplementary Table 4. We found that these CD73-defined groups correlated with tobacco history $(p=0.0194)$; most LUADs from never smoker patients were TL (73\%) and none of them were TN. TL LUADs showed more frequent non-solid histological patterns, while $\mathrm{TH}$ and $\mathrm{TN}$ showed similar proportions of tumors with any-solid histological pattern $(p=0.0003)$ (Table 2). Notably, the CD73-defined groups correlated with most of the immune markers examined (Fig. 3a). TH showed the highest PD-L1 expression in MCs $(p=0.002)$ (Fig. 3b), and significantly higher cell densities of CD3 CD4, CD8, PD-1, FOXP3, Granzyme B, CD45RO, and CD68-positive cells (Fig. 3c). In addition, $22.2 \%$ of tumors evaluated co-expressed CD73 and PD-L1. We did not find significant differences in immune marker expression between TN and TL LUADs. No differences in survival outcomes among the three groups were observed (Supplementary Fig. 5). 
a

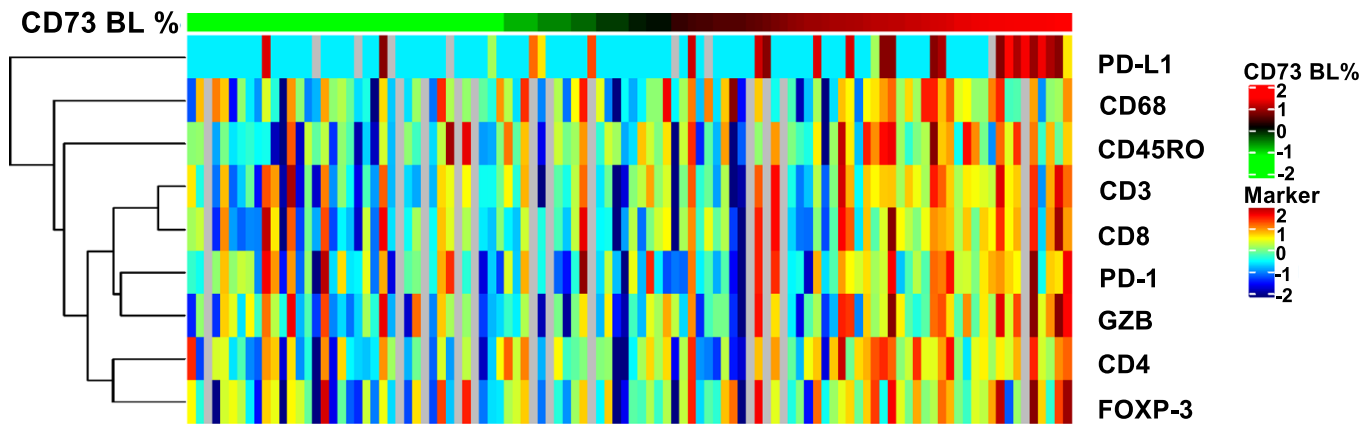

b
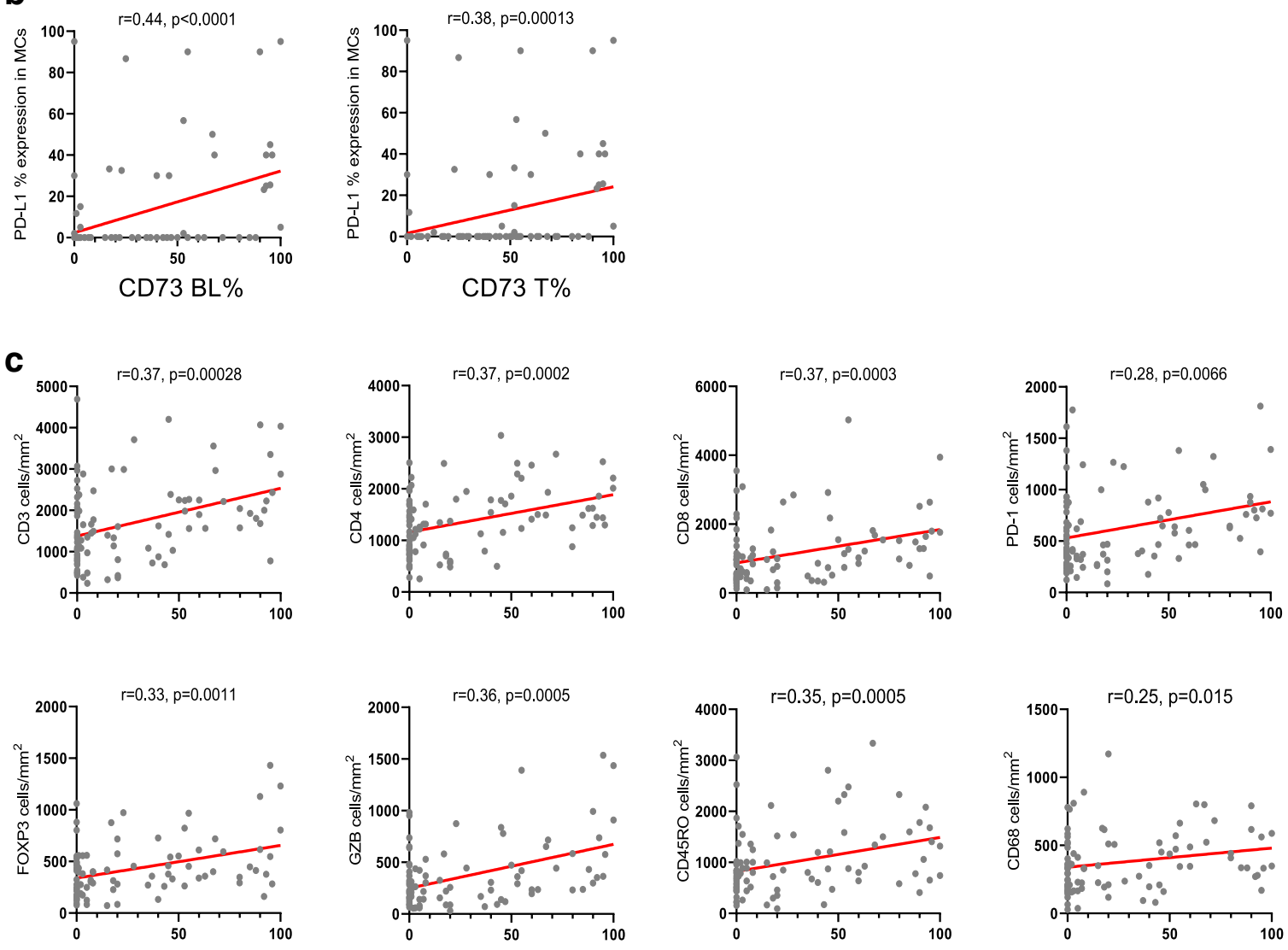

CD73 BL\%

d

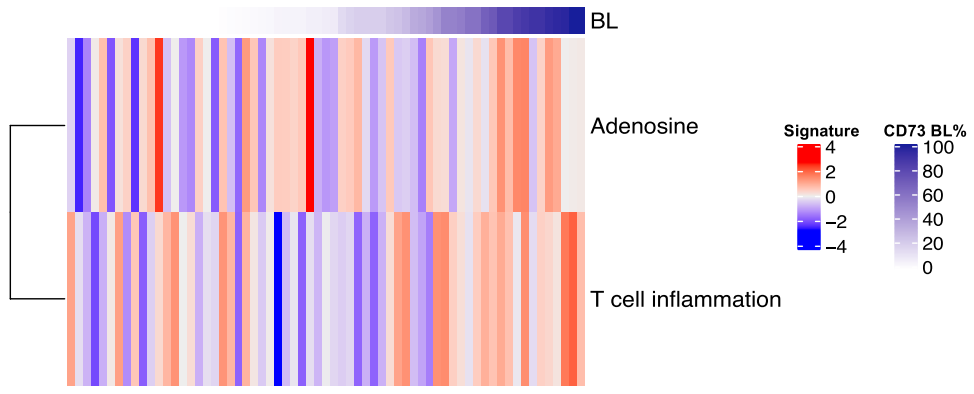


4Fig. 2 Basolateral CD73 expression is associated with higher immune infiltration in lung adenocarcinoma. a Heat map of TAIC densities and PD-L1 (\% of expression) in MCs from 95 LUADs sorted according to BL CD73 expression (red, relatively higher BL CD73 expression; green, lower BL CD73 expression). Rows represent immune marker and columns denote samples (red, relatively higher TAIC density or PD-L1\%; blue, lower TAIC density or PD-L1\%). b Spearman correlation analysis of PD-L1 expression in MCs with BL and T CD73. c Spearman correlation analyses of TAICs ( $y$-axis) with BL CD73 expression ( $x$-axis)

\section{Association of CD73 expression with molecular features}

We then examined the association of CD73 expression with genomic features in LUAD. TH and TN groups showed significantly higher proportion of TP53 mutant tumors $(p=0.0035)$ compared to TL group. Somatic mutation burdens were significantly lower in the TL group compared to TH and TN groups ( $p=0.0018$ ) (Table 2 ). We found that $\mathrm{L}$ negative $\mathrm{CD} 73$ LUADs comprised more STK11 mutant LUADs rates compared to $\mathrm{L}+(p=0.0041)$, although in our cohort, we only have a small number of STK11 mutations $(n=7)$. We did not find associations between $K R A S$ and $E G F R$ mutations with CD73 expression.

\section{Association of CD73 with other markers involved in adenosine generation}

We next interrogated other critical enzymes in the adenosine pathway, namely CD38 and CD39. CD38 expression in malignant cells was found in 20\% (20/98) of LUADs and 18 (89\%) of them co-expressed T CD73. Assessment of CD38 in tumor stroma showed that higher number of $\mathrm{CD} 38^{+}$cells positively associated with the TN group $(p=0.02)$ (Supplementary Fig. 6a). In addition, our immune gene profiling analysis showed that CD38 cell densities in tumor stroma were associated with specific immune cell signatures indicative of T-cell inflammation, cytotoxic T lymphocytes, expanded host immune responses, tumor inflammation (TIS), interferongamma signaling, as well as peripheral T-cell infiltration and M1 macrophage polarization (Supplementary Fig. 6b). In contrast, CD39 expression was not observed in malignant cells in our cohort, and $\mathrm{CD} 39^{+}$cell densities in the tumor stroma did not exhibit significant association with CD73 expression (Supplementary Fig. 6c) or with immune signatures obtained by gene expression analysis.

\section{Discussion}

The immunosuppressive adenosine pathway, in which CD73 plays a critical role, has been proposed as one of the possible mechanisms of resistance to ICI $[9,35,39]$.
The current combination of ICI therapies and anti-CD73 antibodies are attractive therapeutic approaches, and are under evaluation [40] with the aim to improve the outcome in patients with NSCLC that did not response to ICI (monotherapy or combination with chemotherapy). Yet, the role of CD73 in the pathobiology and immune contexture of LUAD is poorly understood. To begin to fill this void, we examined the expression patterns of CD73 in a richly annotated cohort of early stage LUADs in association with various features including clinicopathological, molecular, and immune covariates. We found that CD73 was expressed in a significant fraction (75\%) of LUADs and categorized subsets of LUAD with distinct histological, molecular, and immune features.

In contrast to previous studies that mostly focused on total CD73 expression assessment, we interrogated CD73 in different membrane compartments (basolateral and luminal; BL and L, respectively) of MCs. Our pathological analyses demonstrated that tumors with different CD73 expression patterns exhibited distinct clinicopathological (e.g., histological patterns) and molecular associations, possibly pointing to causal links between CD73 expression or membrane localization and tumor differentiation [9, 41]. This hypothesis is also supported by our finding on progressively increased expression of $C D 73$ across premalignant lung lesions representing different stages in the sequence of LUAD pathogenesis. We also found that the localization of CD73 in cells from well-differentiated LUADs was predominantly luminal, which may as well be related to the physiological protective and mitigating properties of CD73 against inflammation (45). Of note, we found distinct associations between not only the presence or absence of CD73 but also the extent of expression of this antigen with smoking status, molecular features, and immune infiltration (Table 1). Consistent with previous reports [22], our cohort showed that all (100\%) never smoker LUADs exhibited positive CD73 expression when compared to smoker tumors (70\%). However, among positive CD73 tumors, never smoker patients had lower extent of CD73 expression (T Low group), along with more differentiated tumors, less mutation burden, and lower rates of p53 mutation. These results suggest that extensive expression of CD73 in tumors from smoker patients could be in part explained by the higher immune infiltration observed in these tumors. Interestingly, CD73 membrane localization was also predominantly luminal (Supplementary Table 5), while the group with higher extent of CD73 expression, the predominant localization was basolateral. It is reasonable to surmise that CD73, viz., its disparate localization, may have distinct roles in the molecular pathogenesis of smoker and non-smoker LUADs. It is also plausible to suggest that CD73 membrane localization may have important implications on the effectiveness of anti-CD73 therapy. 
Table 2 Clinicopathological and molecular features of LUAD patients grouped based on extent of CD73 expression

\begin{tabular}{|c|c|c|c|c|c|}
\hline Characteristic & $N$ & $\begin{array}{l}\text { T high }(\mathrm{TH}) \\
(26 / 106,25 \%) \\
N(\%)\end{array}$ & $\begin{array}{l}\text { T low (TL) } \\
(53 / 106,50 \%) \\
N(\%)\end{array}$ & $\begin{array}{l}\text { T negative }(\mathrm{TN}) \\
(27 / 106,25 \%) \\
N(\%)\end{array}$ & $p$ values $^{\mathrm{a}}$ \\
\hline \multicolumn{6}{|l|}{ Age } \\
\hline$\leq 65$ & 53 & $17(32 \%)$ & $21(40 \%)$ & $15(28 \%)$ & \multirow[t]{2}{*}{0.2069} \\
\hline$>65$ & 53 & $11(21 \%)$ & $30(57 \%)$ & $12(23 \%)$ & \\
\hline \multicolumn{6}{|l|}{ Sex } \\
\hline Female & 52 & $15(29 \%)$ & $26(50 \%)$ & $11(21 \%)$ & \multirow[t]{2}{*}{0.6116} \\
\hline Male & 54 & $13(24 \%)$ & $25(46 \%)$ & $16(30 \%)$ & \\
\hline \multicolumn{6}{|l|}{ Smoking history } \\
\hline Never & 15 & $4(27 \%)$ & $11(73 \%)$ & $0(0 \%)$ & \multirow[t]{2}{*}{0.0194} \\
\hline Current/former & 91 & $24(26 \%)$ & $40(44 \%)$ & $27(30 \%)$ & \\
\hline \multicolumn{6}{|l|}{ TNM 8th edition } \\
\hline I & 58 & $18(31 \%)$ & $28(48 \%)$ & $12(20 \%)$ & \multirow[t]{3}{*}{0.4244} \\
\hline II & 26 & $4(15 \%)$ & $12(46 \%)$ & $10(39 \%)$ & \\
\hline III & 22 & $6(27 \%)$ & $11(50 \%)$ & $5(23 \%)$ & \\
\hline \multicolumn{6}{|l|}{ Pathological T (8th) } \\
\hline $\mathrm{pT} 1 \mathrm{a}-\mathrm{pT} 2 \mathrm{a}$ & 70 & $19(27 \%)$ & $35(50 \%)$ & $16(23 \%)$ & \multirow[t]{2}{*}{0.6934} \\
\hline $\mathrm{pT} 2 \mathrm{~b}-\mathrm{T} 4$ & 36 & $9(25 \%)$ & $16(44 \%)$ & $11(31 \%)$ & \\
\hline \multicolumn{6}{|l|}{ Pathological N (8th) } \\
\hline N0 & 78 & $22(28 \%)$ & $37(47 \%)$ & $19(24 \%)$ & \multirow[t]{3}{*}{0.9041} \\
\hline N1 & 20 & $5(25 \%)$ & $10(50 \%)$ & $5(25 \%)$ & \\
\hline $\mathrm{N} 2$ & 8 & $1(13 \%)$ & $4(50 \%)$ & $3(37 \%)$ & \\
\hline \multicolumn{6}{|l|}{ Histologic pattern } \\
\hline Any-solid & 46 & $15(32 \%)$ & $14(30 \%)$ & $17(37 \%)$ & \multirow[t]{2}{*}{0.0003} \\
\hline Non-solid & 60 & $11(18 \%)$ & $39(65 \%)$ & $10(17 \%)$ & \\
\hline \multicolumn{6}{|l|}{ Molecular features } \\
\hline$E G F R$ mutated & 15 & $5(33 \%)$ & $9(60 \%)$ & $1(7 \%)$ & \multirow[t]{2}{*}{0.1717} \\
\hline$E G F R$ wild type & 85 & $22(26 \%)$ & $38(45 \%)$ & $25(29 \%)$ & \\
\hline$K R A S$ mutated & 26 & $8(31 \%)$ & $13(50 \%)$ & $5(19 \%)$ & \multirow[t]{2}{*}{0.7039} \\
\hline$K R A S$ wild type & 77 & $20(26 \%)$ & $36(47 \%)$ & $21(27 \%)$ & \\
\hline TP53 mutated & 27 & $11(41 \%)$ & $7(26 \%)$ & $9(33 \%)$ & \multirow[t]{2}{*}{0.0035} \\
\hline TP53 wild type & 36 & $5(14 \%)$ & $24(67 \%)$ & $7(19 \%)$ & \\
\hline STK11 mutated & 7 & $1(14 \%)$ & $2(29 \%)$ & $4(57 \%)$ & \multirow[t]{2}{*}{0.1901} \\
\hline STK11 wild type & 56 & $15(27 \%)$ & $29(52 \%)$ & $12(21 \%)$ & \\
\hline \multicolumn{6}{|l|}{ Mutational burden } \\
\hline Median & 63 & $353(2-955)$ & $154(3-914)$ & $392(33-993)$ & 0.0018 \\
\hline
\end{tabular}

It is important to mention that we observed that tumor BL CD73 expression positively correlated with features of a "hot" immune environment such as PD-L1 and immune cell infiltration rendering the plausible supposition that CD73 immune function may be disparate between BL and L compartments of LUAD cells. Similarly, when we analyzed immune cell densities within LUADs grouped based on CD73 positivity, the TH group displayed elevated PD-L1 and immune cell infiltration compared with the TL and TN groups. It is noteworthy, that CD73 was shown to suppress anti-tumor immunity and promote immune evasion [9, $42,43]$. Thus, given our findings along with the previous reports on CD73 function, it is not unreasonable to suggest that expression of CD73 may underlie inferior response to ICI even in tumors with concomitant high tumoral PD-L1 expression and immune cell infiltration [44, 45]. In line with our results, a previous report demonstrated that high levels of adenosine correlated with elevated infiltration of immune cells, but with a decreased response to ICI [32]. It is intriguing to suppose that targeting CD73 may enhance antitumor immunity, particularly in tumors with high levels of CD73, as well as augment the effect of ICI. Indeed, targeting CD73 was shown to skew the immune TME to a more antitumor phenotype in preclinical models $[46,47]$. In separate context, our findings also suggest that targeting CD73 may help augment anti-tumor immunity in LUADs with low yet 
a
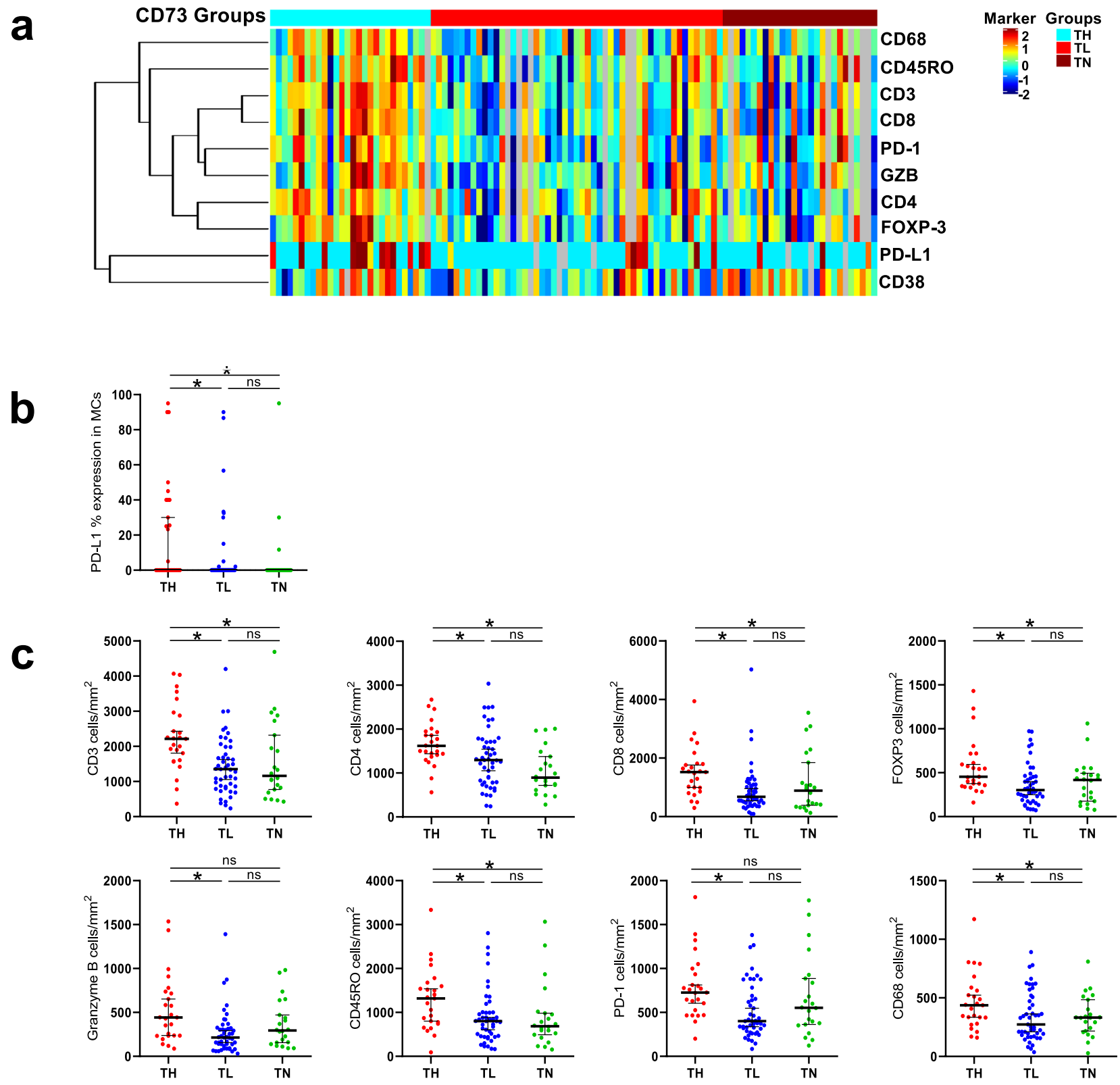

Fig. 3 Extent of CD73 expression defines groups of lung adenocarcinoma with disparate tumor immune infiltration. a Heat map of TAIC densities and PD-L1 (\% of expression) in MCs of 95 LUADs grouped based on the extent of CD73 expression (TH, TL, and TN). Rows represent immune markers and columns denote samples (red, relatively

positive CD73, and which we find here to exhibit a relatively "cold" immune contexture [48]. Of note, we found that a fraction of LUADs that were CD73 negative displayed abundant expression of CD38 concomitant with a muted host immune response, suggesting redundant activation of the non-canonical adenosine pathway $[9,11]$ in these tumors and their potential tractability by agents that target this pathway. Our study points to the potential role of CD73, and higher TAIC density or PD-L1\%; blue, relatively lower TAIC density or PD-L1\%). b Plots showing PD-L1\% expression among the TH, TL, and TN groups. c) Plots showing TAIC densities among the TH, TL, and TN groups $\left({ }^{*} p<0.05\right.$ based on the Kruskal-Wallis test, $n . s$. not significant, bars correspond to median values $\pm 95 \% \mathrm{CI}$ )

other members of the adenosine signaling pathway, as potential mechanisms of tumor immune evasion and resistance to ICI, thus providing additional rationale for propagating anti-CD73 antibodies in new combinatorial immunotherapeutic regimens. As mentioned before, we found that differential (e.g., BL vs. L) CD73 localization was associated with distinct clinicopathological and molecular features in LUAD. It is intriguing to propose that in-depth assessment 
of CD73 expression along with its membrane localization will provide comprehensive assessment of patients who may benefit from agents targeting this immune marker.

Our study is not without limitations. It is important to mention that we interrogated tissue microarrays of LUAD, with these arrays typically harboring relatively small tissue cores which may bring about increased tumor and, thus, immune marker heterogeneity and under-representation of luminal structures of adenocarcinomas-thus warranting future studies probing CD73 in whole tissue specimens. It is also noteworthy, given our study design and goals, that our cohort was primarily comprised of resectable early stage tumors with, thus, under-representation of relatively more advanced (e.g., metastatic) LUADs. In this context, our study is unable to ascertain relative patterns of CD73 expression (and localization), along with features of host anti-tumor immunity and immune evasion, between early stage and more advanced LUADs. Since mechanisms of host immune evasion by the tumor, along with genomic and mutational complexity, are expectantly more pronounced in advanced-stage tumors, future studies are warranted to fully probe $\mathrm{CD} 73$, and other members of the adenosine pathway, along the continuum of different stages (e.g., early, local/ oligometastatic to distant metastatic) in LUAD. Furthermore, the expression patterns of CD73 in patients who have received ICI, preoperatively or in the advanced setting, are not yet discerned. It also cannot be neglected that our study is retrospective in nature and comprises a cohort of limited number of patients and from a single center warranting validation of our findings by external cohorts. Additionally, future studies are warranted that further probe mechanisms involving CD73 expression and its interaction with host immune responses in LUAD. It is important to note that, unlike earlier work [22], we did not find associations between CD73 with clinical outcome and EGFR mutation status. This discrepancy may be attributable to the disparate patient molecular and clinicopathological profiles in our cohort compared to those earlier studies that focused on East Asian patients [22]. Due to the lack of tissue availability, the analysis of $C D 73$ gene expression in AAH, MIA, AIS, LUAD, and normal lung tissue was not validated by IHC. Nonetheless, our study provides new and comprehensive insights into diverse patterns of CD73 expression and localization, in association with genomic, immune, and clinical features, in early stage LUAD, thus offering a roadmap in the future to interrogate the role of CD73 expression in immunotherapy and/or response to ICI.

In conclusion, we comprehensively surveyed the expression, abundance, and membrane tumor localization of CD73 in early stage LUAD, and found that this immune marker with distinct clinicopathological, molecular, and immune characteristics. Our findings on increased expression of the immune evasion mediator CD73 in LUADs with elevated
PD-L1 and immune cell infiltration offer potential insight into why some patients with augmented immune response still respond poorly or modestly to ICI. Our data also provide the plausible rationale for exploring immunotherapeutic regimens consisting of anti-CD73 antibodies in combination with ICI.

Supplementary Information The online version contains supplementary material available at https://doi.org/10.1007/s00262-020-02820-4.

Acknowledgements The author would like to thank the laboratory members of the Translational Molecular Pathology Immune-Profiling Laboratory (TMP-IL), especially Lakshmi Kakarala, Mei Jiang, Auriole Tamegnon, Jocelyn Coronel, and Jianling Zhou for their technical assistance.

Author contributions Study concept and design: LS, CB, and IW; Develop of methodology: PR, RS, BM, WL, and LS; Data acquisition: RS, DL, PV, HD, DD, CB, KG, AW, EP, DR, BS, NK, CM, AV, TC, JH, DG, JZ, and LS; Data interpretation: PR, CB, HK, and LS; Statistical analysis: JZ, LD, and JL; Writing of the manuscript: PR, CB, HK, IW, and LS; Edition and approval of the manuscript: all authors.

Funding This work was partially supported by the NCI Specialized Program of Research Excellence (SPORE) in Lung Cancer (P50CA70907), by the Cancer Prevention and Research Institute of Texas Multi-Investigator Research Award (CPRIT MIRA) grant (RP160668) at The University of Texas M.D. Anderson Cancer Center, and by SEOM (Sociedad Española de Oncología Médica).

Data availability Data are available upon reasonable request. The data that support the findings of our study are available upon request from the corresponding author. The data are not publicly available due to privacy or ethical restrictions.

Code availability Not applicable.

\section{Compliance with ethical standards}

\section{Conflict of interest None.}

Ethical approval This study was approved by the MD Anderson Institutional Review Board and was conducted according to the principles of the Helsinki Declaration.

Open Access This article is licensed under a Creative Commons Attribution 4.0 International License, which permits use, sharing, adaptation, distribution and reproduction in any medium or format, as long as you give appropriate credit to the original author(s) and the source, provide a link to the Creative Commons licence, and indicate if changes were made. The images or other third party material in this article are included in the article's Creative Commons licence, unless indicated otherwise in a credit line to the material. If material is not included in the article's Creative Commons licence and your intended use is not permitted by statutory regulation or exceeds the permitted use, you will need to obtain permission directly from the copyright holder. To view a copy of this licence, visit http://creativecommons.org/licenses/by/4.0/. 


\section{References}

1. Siegel RL, Miller KD, Jemal A (2020) Cancer statistics, 2020. CA Cancer J Clin 70:7-30. https://doi.org/10.3322/caac.21590

2. Borghaei H, Paz-Ares L, Horn L et al (2015) Nivolumab versus docetaxel in advanced nonsquamous non-small-cell lung cancer. N Engl J Med 373:1627-1639. https://doi.org/10.1056/NEJMo a1507643

3. Reck M, Rodríguez-Abreu D, Robinson AG et al (2016) Pembrolizumab versus chemotherapy for PD-L1-positive non-smallcell lung cancer. N Engl J Med 375:1823-1833. https://doi. org/10.1056/NEJMoa1606774

4. Gandhi L, Rodríguez-Abreu D, Gadgeel S et al (2018) Pembrolizumab plus chemotherapy in metastatic non-small-cell lung cancer. N Engl J Med. https://doi.org/10.1056/NEJMoa1801005

5. Gettinger S, Horn L, Jackman D et al (2018) Five-year followup of Nivolumab in previously treated advanced non-small-cell lung cancer: results from the CA209-003 study. J Clin Oncol 003:2017770412. https://doi.org/10.1200/JCO.2017.77.0412

6. Forde PM, Chaft JE, Smith KN et al (2018) Neoadjuvant PD-1 blockade in resectable lung cancer. N Engl J Med 378:1976-1986. https://doi.org/10.1056/NEJMoa1716078

7. Antonia SJ, Villegas A, Daniel D et al (2017) Durvalumab after chemoradiotherapy in stage III non-small-cell lung cancer. N Engl J Med 377:1919-1929. https://doi.org/10.1056/NEJMoa1709937

8. Gao ZW, Dong K, Zhang HZ (2014) The roles of CD73 in cancer. Biomed Res Int 2014:460654. https://doi.org/10.1155/2014/46065 4

9. Allard B, Allard D, Buisseret L, Stagg J (2020) The adenosine pathway in immuno-oncology. Nat Rev Clin Oncol. https://doi. org/10.1038/s41571-020-0382-2

10. Fridman WH, Zitvogel L, Sautès-Fridman C, Kroemer G (2017) The immune contexture in cancer prognosis and treatment. Nat Rev Clin Oncol 14:717-734. https://doi.org/10.1038/nrcli nonc.2017.101

11. Horenstein AL, Chillemi A, Zaccarello G et al (2013) A CD38/ CD203a/CD73 ectoenzymatic pathway independent of CD39 drives a novel adenosinergic loop in human T lymphocytes. Oncoimmunology 2:e26246. https://doi.org/10.4161/onci.26246

12. de Andrade MP, Coutinho-Silva R, Savio LEB (2017) Multifaceted effects of extracellular adenosine triphosphate and adenosine in the tumor-host interaction and therapeutic perspectives. Front Immunol 8:1526. https://doi.org/10.3389/fimmu.2017.01526

13. Regateiro FS, Cobbold SP, Waldmann H (2013) CD73 and adenosine generation in the creation of regulatory microenvironments. Clin Exp Immunol 171:1-7. https://doi.org/10.111 1/j.1365-2249.2012.04623.x

14. Beavis PA, Divisekera U, Paget C et al (2013) Blockade of A 2A receptors potently suppresses the metastasis of $\mathrm{CD} 73^{+}$tumors. Proc Natl Acad Sci 110:14711-14716. https://doi.org/10.1073/ pnas. 1308209110

15. Antonioli L, Blandizzi C, Pacher P, Haskó G (2013) Immunity, inflammation and cancer: a leading role for adenosine. Nat Rev Cancer 13:842-857. https://doi.org/10.1038/nrc3613

16. Chen S, Wainwright DA, Wu JD et al (2019) CD73: an emerging checkpoint for cancer immunotherapy. Immunotherapy 11:983997. https://doi.org/10.2217/imt-2018-0200

17. Chen DS, Mellman I (2013) Oncology meets immunology: the cancer-immunity cycle. Immunity 39:1-10. https://doi. org/10.1016/j.immuni.2013.07.012

18. Cogdill AP, Andrews MC, Wargo JA (2017) Hallmarks of response to immune checkpoint blockade. Br J Cancer 117:1-7. https://doi.org/10.1038/bjc.2017.136
19. Marshall HT, Djamgoz MBA (2018) Immuno-oncology: emerging targets and combination therapies. Front Oncol 8:1-29. https://doi. org/10.3389/fonc.2018.00315

20. Young A, Ngiow SF, Barkauskas DS et al (2016) Co-inhibition of CD73 and A2AR adenosine signaling improves antitumor immune responses. Cancer Cell 30:391-403. https://doi. org/10.1016/j.ccell.2016.06.025

21. Stagg J, Divisekera U, McLaughlin N et al (2010) Anti-CD73 antibody therapy inhibits breast tumor growth and metastasis. Proc Natl Acad Sci 107:1547-1552. https://doi.org/10.1073/ pnas.0908801107

22. Inoue $\mathrm{Y}$, Yoshimura K, Kurabe $\mathrm{N}$ et al (2017) Prognostic impact of CD73 and A2A adenosine receptor expression in non-small-cell lung cancer. Oncotarget 8:8738-8751. https://doi.org/10.18632/ oncotarget. 14434

23. Kudo Y, Haymaker C, Zhang J et al (2019) Suppressed immune microenvironment and repertoire in brain metastases from patients with resected non-small-cell lung cancer. Ann Oncol Off J Eur Soc Med Oncol 30:1521-1530. https://doi.org/10.1093/annonc/mdz20 7

24. Solis LM, Behrens C, Dong W et al (2010) Nrf2 and Keap1 abnormalities in non-small cell lung carcinoma and association with clinicopathologic features. Clin Cancer Res 16:3743-3753. https ://doi.org/10.1158/1078-0432.CCR-09-3352

25. Rami-Porta R, Asamura H, Travis WD, Rusch VW (2017) Lung cancer-major changes in the American joint committee on cancer eighth edition cancer staging manual. CA Cancer J Clin. 67:138 155. https://doi.org/10.3322/caac. 21390

26. Solis LM, Behrens C, Raso MG et al (2012) Histologic patterns and molecular characteristics of lung adenocarcinoma associated with clinical outcome. Cancer 118:2889-2899. https://doi. org/10.1002/cncr.26584

27. Kadara H, Choi M, Zhang J et al (2017) Whole-exome sequencing and immune profiling of early-stage lung adenocarcinoma with fully annotated clinical follow-up. Ann Oncol Off J Eur Soc Med Oncol 28:75-82. https://doi.org/10.1093/annonc/mdw436

28. Ayers M, Lunceford J, Nebozhyn M et al (2017) IFN- $\gamma$-related mRNA profile predicts clinical response to PD-1 blockade. J Clin Invest 127:2930-2940. https://doi.org/10.1172/JCI91190

29. Spranger S, Bao R, Gajewski TF (2015) Melanoma-intrinsic $\beta$-catenin signalling prevents anti-tumour immunity. Nature 523:231-235. https://doi.org/10.1038/nature14404

30. Jiang P, Gu S, Pan D et al (2018) Signatures of T cell dysfunction and exclusion predict cancer immunotherapy response. Nat Med 24:1550-1558. https://doi.org/10.1038/s41591-018-0136-1

31. Hwang S, Kwon A-Y, Jeong J-Y et al (2020) Immune gene signatures for predicting durable clinical benefit of anti-PD-1 immunotherapy in patients with non-small cell lung cancer. Sci Rep 10:643. https://doi.org/10.1038/s41598-019-57218-9

32. Sidders B, Zhang P, Goodwin K et al (2020) Adenosine signaling is prognostic for cancer outcome and has predictive utility for immunotherapeutic response. Clin Cancer Res 26:2176-2187. https://doi.org/10.1158/1078-0432.CCR-19-2183

33. Danaher P, Warren S, Lu R et al (2018) Pan-cancer adaptive immune resistance as defined by the tumor inflammation signature (TIS): results from The Cancer Genome Atlas (TCGA). J Immunother Cancer 6:63. https://doi.org/10.1186/s40425-018-0367-1

34. Qi Z, Wang L, Desai K et al (2019) Reliable gene expression profiling from small and hematoxylin and eosin-stained clinical formalin-fixed, paraffin-embedded specimens using the HTG edgeseq platform. J Mol Diagn 21:796-807. https://doi.org/10.1016/j. jmoldx.2019.04.011

35. Chen L, Diao L, Yang Y et al (2018) CD38-mediated immunosuppression as a mechanism of tumor cell escape from PD-1/ PD-11 blockade. Cancer Discov 8:1156-1175. https://doi. org/10.1158/2159-8290.CD-17-1033 
36. Lantuejoul S, Sound-Tsao M, Cooper WA et al (2020) PD-L1 testing for lung cancer in 2019: perspective from the IASLC Pathology Committee. J Thorac Oncol 15:499-519. https://doi. org/10.1016/j.jtho.2019.12.107

37. Parra ER, Behrens C, Rodriguez-Canales J et al (2016) Image analysis-based assessment of pd-11 and tumor-associated immune cells density supports distinct intratumoral microenvironment groups in non-small cell lung carcinoma patients. Clin Cancer Res 22:6278-6289. https://doi.org/10.1158/1078-0432.CCR-15-2443

38. Parra ER, Villalobos P, Zhang J et al (2018) Immunohistochemical and image analysis-based study shows that several immune checkpoints are co-expressed in non-small cell lung carcinoma tumors. J Thorac Oncol 13:779-791. https://doi.org/10.1016/j. jtho.2018.03.002

39. Hay CM, Sult E, Huang Q et al (2016) Targeting CD73 in the tumor microenvironment with MEDI9447. Oncoimmunology 5:e1208875. https://doi.org/10.1080/2162402X.2016.1208875

40. NIH-U.S. National Library of Medicine (2019) ClinicalTrials. gov. https://clinicaltrials.gov/ct2/results?cond=Lung+Cance $\mathrm{r} \&$ term $=\mathrm{CD} 73 \&$ cntry $=\&$ state $=\&$ city $=\&$ dist $=\&$ Search $=$ Search

41. Pervez S, Arshad S, Abro B (2018) HER2 basolateral versus circumferential IHC expression is dependent on polarity and differentiation of epithelial cells in gastric/GE adenocarcinoma. Patholog Res Int 2018:6246493. https://doi.org/10.1155/2018/6246493

42. Vigano S, Alatzoglou D, Irving M et al (2019) Targeting adenosine in cancer immunotherapy to enhance T-Cell function. Front Immunol 10:925. https://doi.org/10.3389/fimmu.2019.00925

43. Chen S, Fan J, Zhang M et al (2019) CD73 expression on effector $\mathrm{T}$ cells sustained by TGF- $\beta$ facilitates tumor resistance to
anti-4-1BB/CD137 therapy. Nat Commun 10:150. https://doi. org/10.1038/s41467-018-08123-8

44. Teng F, Meng X, Kong L, Yu J (2018) Progress and challenges of predictive biomarkers of anti PD-1/PD-L1 immunotherapy: a systematic review. Cancer Lett 414:166-173. https://doi. org/10.1016/j.canlet.2017.11.014

45. Minor M, Alcedo KP, Battaglia RA, Snider NT (2019) Cell typeand tissue-specific functions of ecto-5'-nucleotidase (CD73). Am J Physiol Cell Physiol. https://doi.org/10.1152/ajpcell.00285.2019

46. Beavis PA, Milenkovski N, Henderson MA et al (2015) Adenosine receptor $2 \mathrm{~A}$ blockade increases the efficacy of anti-PD-1 through enhanced antitumor T-cell responses. Cancer Immunol Res 3:506-517. https://doi.org/10.1158/2326-6066.CIR-14-0211

47. Perrot I, Michaud H-A, Giraudon-Paoli M et al (2019) Blocking antibodies targeting the CD39/CD73 immunosuppressive pathway unleash immune responses in combination cancer therapies. Cell Rep 27:2411-2425.e9. https://doi.org/10.1016/j.celre p.2019.04.091

48. Mazzarella L, Duso BA, Trapani D et al (2019) The evolving landscape of "next-generation" immune checkpoint inhibitors: a review. Eur J Cancer 117:14-31. https://doi.org/10.1016/j. ejca.2019.04.035

Publisher's Note Springer Nature remains neutral with regard to jurisdictional claims in published maps and institutional affiliations.

\section{Authors and Affiliations}

\section{Pedro Rocha ${ }^{1,2} \cdot$ Ruth Salazar $^{1}$. Jiexin Zhang ${ }^{3} \cdot$ Debora Ledesma $^{1}$ - Jose L. Solorzano ${ }^{1} \cdot$ Barbara Mino ${ }^{1}$. Pamela Villalobos ${ }^{1} \cdot$ Hitoshi Dejima ${ }^{1} \cdot$ Dzifa Y. Douse ${ }^{1} \cdot$ Lixia Diao $^{3} \cdot$ Kyle Gregory Mitchell $^{4} \cdot$ Xiuning Le $^{4}$. Jianjun Zhang ${ }^{4} \cdot$ Annikka Weissferdt $^{6} \cdot$ Edwin Parra-Cuentas $^{1} \cdot$ Tina Cascone $^{4} \cdot$ David C. Rice $^{5} \cdot$ Boris Sepesi $^{4}$. Neda Kalhor ${ }^{6}$. Cesar Moran ${ }^{6}$. Ara Vaporciyan ${ }^{5}$. John Heymach ${ }^{4}$. Don L. Gibbons ${ }^{4}$. J. Jack Lee ${ }^{3} \cdot$ Humam Kadara ${ }^{1}$. Ignacio Wistuba ${ }^{1,4} \cdot$ Carmen Behrens $^{4} \cdot$ Luisa Maren Solis ${ }^{1}$ (1)}

1 Department of Translational Molecular Pathology, The University of Texas MD Anderson Cancer Center, 2130 West Holcombe Boulevard, Houston, TX 77030, USA

2 Universidad de Barcelona, Barcelona, Spain

3 Department of Bioinformatics and Comp Biology, The University of Texas MD Anderson Cancer Center, Houston, TX, USA
4 Thoracic/Head and Neck Medical Oncology, The University of Texas MD Anderson Cancer Center, Houston, TX, USA

5 Thoracic and Cardiovascular Surgery, The University of Texas MD Anderson Cancer Center, Houston, TX, USA

6 The University of Texas MD Anderson Cancer Center, Houston, TX, USA 